\title{
Magnetohydrodynamic waveguide/resonator for Pc3 ULF pulsations at cusp latitudes
}

\author{
V. Pilipenko ${ }^{1}$, E. Fedorov ${ }^{1}$, N. Mazur ${ }^{1}$, M. J. Engebretson ${ }^{2}$, and W. J. Hughes ${ }^{3}$ \\ ${ }^{1}$ Institute of the Physics of the Earth, Moscow 123810, Russia \\ ${ }^{2}$ Department of Physics, Augsburg College, Minneapolis, MN 55454, U.S.A. \\ ${ }^{3}$ Center for Space Physics, Boston University, Boston, MA 02215, U.S.A.
}

(Received April 9, 1999; Accepted June 3, 1999)

\begin{abstract}
We present a possible theoretical model for the generation of travelling Alfven waves by external compressional disturbances in a two-dimensional inhomogeneous plasma. The transformation of a compressional wave mode into Alfven waves guided by the magnetic field is considered in a region with decreased Alfven velocity. This region represents, on the one hand, a waveguide for compressional oscillations, and on the other hand, a resonator with opaque boundaries for Alfven waves. The wave transformation is most effective in a resonant case, when the frequency of a compressional mode approaches the local Alfven frequency of a resonator. This model is applied to the interpretation of Pc3 observations in the cusp region. The mechanism can ensure the selective amplification of magnetosheath turbulence in the Pc3 band in the high-latitude cusp.
\end{abstract}

\section{Introduction}

The dayside cusp/cleft region is characterized by an increase in the intensity of ULF wave activity over a wide frequency range (Olson, 1986; Glassmeier, 1989). However, the Pc3 band (20-100 mHz) is especially prominent beneath the cusp as compared with the background level (Engebretson et al., 1986; Lepidi et al., 1996). Several types of fluctuations in the nominal Pc3 band have been noted near the footprint of the cusp. The first and most common one is noise-like wideband fluctuations, classified as Pi1 pulsations (Engebretson et al., 1995). Another one is the proper Pc3: narrow-band short-lived wavelets (Engebretson et al., 1986; Olson and Fraser, 1994). Engebretson et al. (1995) indicated that the cusp-associated Pc3 pulsations appearing in dynamic FFT spectrograms are band-limited, but can often be masked by strong Pil activity. The intense Pil pulsations dominate at high solar wind velocities, while Pc3 can be observed at cusp latitudes when the solar wind velocity is low or moderate (Engebretson et al., 1995). An intermediate group is composed of band-limited Pc3 pulsations. The occurrence of narrow-band and band-limited Pc3 is found to be controlled by the cone-angle of the IMF, whereas their central frequency is related to IMF magnitude (Engebretson et al., 1986, 1989; Plyasova-Bakounina et al., 1986). However, a linear relationship between the frequency of cusp-associated ULF waves and hourly averages of the IMF magnitude was noted by Wolfe et al. (1990) only for ULF waves above a cutoff frequency about $30 \mathrm{mHz}$.

The variety of the observed types of ULF wave activity in the nominal Pc3 frequency band in the region of cusp projection is caused by inputs of different generation mech-

Copy right (c) The Society of Geomagnetism and Earth, Planetary and Space Sciences (SGEPSS); The Seismological Society of Japan; The Volcanological Society of Japan; The Geodetic Society of Japan; The Japanese Society for Planetary Sciences. anisms. Most probably, as Engebretson et al. (1991a) proposed, wide-band Pi1 pulsations and some Pc3 pulsations are closely related to the fluxes of precipitating particles in the cusp region. Engebretson et al. (1994) showed quantitatively that cusp Pc3 magnetic pulsations can be produced by the modulated precipitation of energetic particles. This idea, though not thoroughly theoretically justified yet, explains many interrelationships between auroral, riometric and ULF magnetic observations in the cusp region.

The actual interrelationships between ULF signals and particle modulation have not been revealed so far. This modulation may be an intrinsic property of intense particle fluxes and develop in a spontaneous way, whereas magnetic noise would be an image of the irregular character of the overhead precipitation. Otherwise, the particle modulation may be induced by external ULF wave activity in the high-altitude cusp. The source of the Pc3 activity in the cusp might be the turbulent hydromagnetic noise in the magnetosheath, penetrating into the magnetosphere (Engebretson et al., 1989, 1991a). Simultaneous observations in the upstream solar wind, subsolar magnetosheath, and outer equatorial magnetosphere that focus on Pc3-4 pulsations (Engebretson et al., 1991b; Lin et al., 1991) showed that during times the IMF geometry supports the generation of upstream waves in the foreshock region, the magnetosheath is characterized by intense broadband turbulence in the magnetic field (with no recognizable sinusoids anywhere near Pc3-4 frequencies) and with large variations in all bulk plasma parameters and greatly increased populations of energized plasma.

The occurrence of narrow-band Pc3 signals on the ground at cusp latitudes implies the operation of some natural mechanism of frequency-selective noise filtering/amplification at cusp latitudes. These signals are unlikely to be produced by direct penetration of magnetosheath turbulence to the iono- 
sphere, as the spectral content of hydromagnetic fluctuations in the magnetosheath downstream of a quasi-parallel shock takes the form of broadband noise, with no distinct peaks (Engebretson et al., 1991b; Le and Russell, 1994).

It is widely accepted that, at middle latitudes, the narrowband spectral structure of Pc3 waves is formed owing to resonant features of the magnetospheric Alfven resonator. The magnetospheric resonator is formed along geomagnetic field lines, and terminated with conductive ionospheres (Southwood, 1974). At cusp latitudes, however, this type of resonator is can hardly be operative. At near-cusp closed field lines the fundamental eigenfrequency is about $T_{\mathrm{A}} \simeq 5-$ $10 \mathrm{~min}$, so the possibility of excitation of standing Alfven harmonics with numbers $n=T_{\mathrm{A}} / T_{\mathrm{Pc} 3} \simeq 10-20$ appears to be unrealistic. Further, in contrast with ULF satellite observations in the inner magnetosphere, the cusp-related Pc3 variations show little evidence of discrete harmonics.

Moreover, compressional disturbances penetrating through the magnetopause in the polar cusp region from the magnetosheath apparently cannot propagate deep into the magnetosphere: ray-tracing of Pc3 compressional waves in multi-component plasma by Zhang et al. (1993) showed that waves in the Pc3 range are trapped in the region of the highlatitude cusp.

Here we suggest an alternative hypothesis for the mechanism of natural band-filtering near the high-latitude cusp. We propose that in the region of the high-altitude cusp (entry layer) compressional wide-band noise may convert into guided propagating Alfven waves, leaking along field lines toward the ionosphere. This transformation has a resonant character, which forms a band-limited spectrum of ULF waves near the geomagnetic projection of the cusp.

\section{Emission of Alfven Waves from a MHD Wave- guide}

Let us consider linear oscillations of a cold $(\beta \ll 1)$ plasma embedded in a magnetic box. Accounting for a finite plasma pressure is essential for the consideration of small-scale kinetic effects or a slow magnetosonic mode, but it does not influence the spectral features of fast magnetosonic and Alfven modes. The $z$ axis of a Cartesian coordinate system is assumed to be oriented along a homogeneous magnetic field $\mathbf{B}_{0}=B_{0} \mathbf{e}_{z}$. Though in a real system the spatial distribution of $V_{\mathrm{A}}$ is produced by variations of both the magnetic field and the plasma density, for simplicity we assume the inhomogeneity of an Alfven velocity to be produced by the plasma density inhomogeneity only, i.e. $\rho_{0}=\rho_{0}(x, z)$. In contrast with the well-known theory of a magnetospheric field line resonator at mid-latitudes (Southwood, 1974) our suggested mechanism assumes the excitation of propagating (non-standing) Alfven waves. A sketch of this model is depicted in Fig. 1.

The disturbed electromagnetic field is presented as a sum of partial Alfven $\left(\mathbf{E}^{(a)}, \mathbf{B}^{(a)}\right)$ and compressional $\left(\mathbf{E}^{(s)}, \mathbf{B}^{(s)}\right)$ waves. This decomposition is convenient to perform with the help of potentials for Alfven $(\varphi)$ and compressional $(\psi)$ disturbances, as follows

$$
\begin{array}{ll}
\mathbf{E}^{(a)}=-\nabla_{\perp} \varphi, & \partial_{t} \mathbf{B}^{(a)}=c\left[\mathbf{e}_{z} \times \nabla_{\perp}\right] \partial_{z} \varphi, \\
\mathbf{E}^{(s)}=\left[\mathbf{e}_{z} \times \nabla_{\perp}\right] \psi, & \partial_{t} \mathbf{B}^{(s)}=c\left(\nabla_{\perp} \partial_{z} \psi-\left(\nabla_{\perp}^{2} \psi\right) \mathbf{e}_{z}\right)(1 \mathrm{~b})
\end{array}
$$
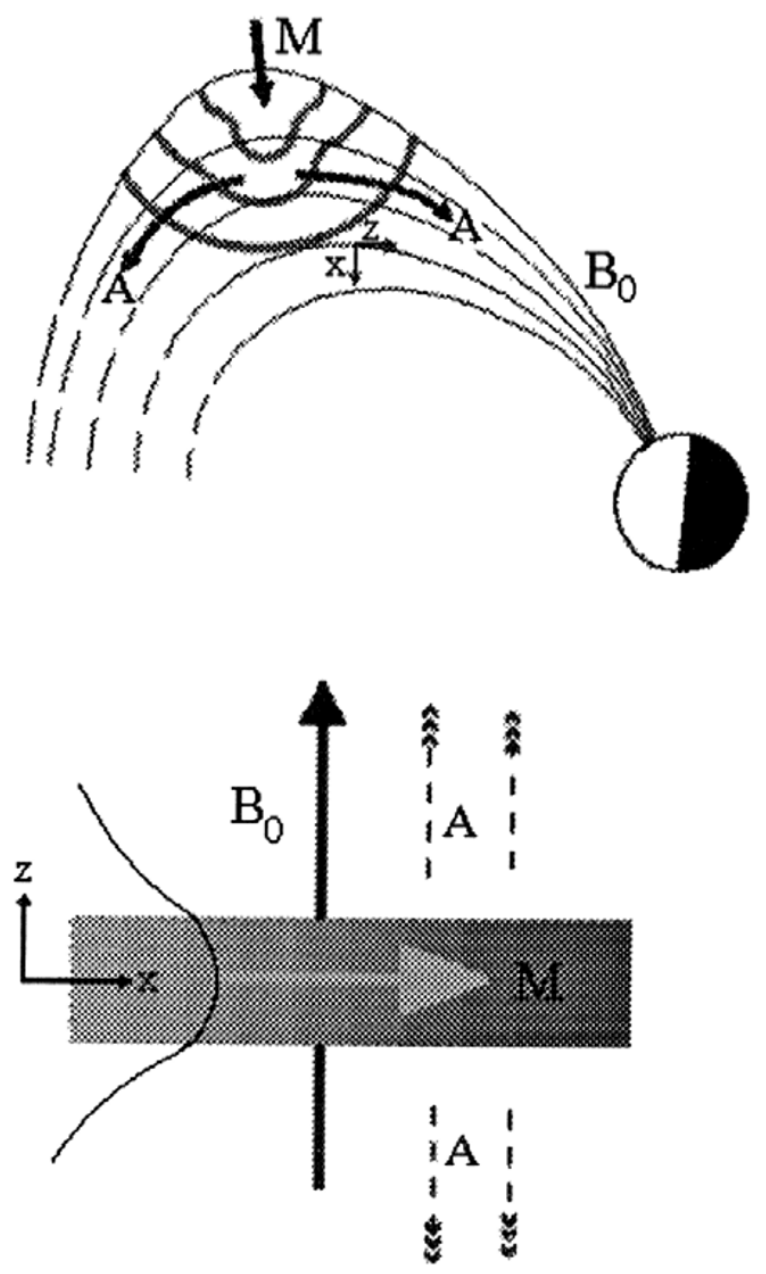

Fig. 1. (upper). Schematic plot of the transformation of magnetosheath disturbances (M) into propagating Alfven waves (A) in a cusp-related MHD waveguide. Grey lines stand for the isolines of an Alfven velocity; (bottom). The model of a plasma waveguide, where a compressional mode (M) (its field-aligned distribution is depicted near the coordinate axis) converts into propagating away Alfven waves (A).

The differential operators in (1) are $\nabla_{\perp}=\left(\partial_{x}, i k_{y}, 0\right),\left[\mathbf{e}_{z} \times\right.$ $\left.\nabla_{\perp}\right]=\left(-i k_{y}, \partial_{x}, 0\right)$, and $\nabla_{\perp}^{2}=\partial_{x x}-k_{y}^{2}$. The potential $\varphi$ is proportional to the field-aligned current $j_{z}$ transmitted by an MHD disturbance, as follows from $\partial_{t} j_{z}=\left(c^{2} / 4 \pi\right) \nabla_{\perp}^{2} \partial_{z} \varphi$. The potential $\psi$ characterizes magnetic field compression, that is, $\partial_{t} B_{z}^{(s)}=-c \nabla_{\perp}^{2} \psi$. Because this model system is homogeneous along the $y$ axis, we consider only a particular harmonic $\exp \left(i k_{y} y-i \omega t\right)$. For the complex Fourier harmonic of potentials $\varphi$ and $\psi$ the system of Maxwell's and ideal MHD equations reduces to

$$
\begin{aligned}
\partial_{x} \hat{L}_{\mathrm{A}} \partial_{x} \varphi-k_{y}^{2} \hat{L}_{\mathrm{A}} \varphi & =-i k_{y} g \psi, \\
\partial_{x} \hat{L}_{\mathrm{M}} \partial_{x} \psi-k_{y}^{2} \hat{L}_{\mathrm{M}} \psi & =i k_{y} g \varphi .
\end{aligned}
$$

Here $\hat{L}_{\mathrm{A}}=\partial_{z z}+k_{\mathrm{A}}^{2}$ denotes the Alfven operator, $\hat{L}_{\mathrm{M}}=$ $\nabla_{\perp}^{2}+\partial_{z z}+k_{\mathrm{A}}^{2}$ is the "magnetosonic" operator, and $k_{\mathrm{A}}(x, z)=$ $\omega / V_{\mathrm{A}}(x, z)$ is an Alfven wave number. The factor $g(x, z)=$ $\partial_{x} k_{\mathrm{A}}^{2}(x, z)$ is due to the transverse inhomogeneity of a system and determines the coupling between modes.

Below we consider the mechanism of Alfven wave generation by a compressional mode, which propagates in a two- 
dimensionally inhomogeneous plasma. It is assumed that the field aligned distribution of $V_{A}(z)$ has a symmetrical minimum at $z=0$, thus forming a waveguide for a compressional mode.

The common field line resonance theory (e.g., Fedorov et $a l ., 1995)$ has a local character, i.e., it describes the wave field only in the vicinity of a resonant shell, where $\hat{L}_{\mathrm{A}} \varphi \rightarrow 0$, and can provide exact magnitudes of the excitation coefficients only with the help of additional numerical calculations. However, for a gradual variation of an Alfven velocity $V_{\mathrm{A}}(x)$ or for small azimuthal wave numbers $k_{y}$ an asymptotic theory can be constructed, and the fast compressional mode transformation into Alfven waves can be described analytically throughout the system. So, we assume that the variation of $V_{\mathrm{A}}$ along the $x$ axis (across the geomagnetic field) is smooth. One can take into account this dependence explicitly by introducing the small parameter $\varepsilon=$ (scale of the disturbance variation)/(scale of the plasma system inhomogeneity). Then the standard WKB approach can be applied. The details of the analytical formalism and more general relationships can be found in Fedorov et al. (1998).

The interaction between compressional and Alfven waves is weak when the coupling factor $\varepsilon k_{y}$ is small. In a zerothorder approximation over this parameter these modes do not interact with each other and can be described by decoupled Eqs. (2a) and ( $2 \mathrm{~b}$ ) with vanishing right-hand sides. In this case (2a) reduces to the equation for small-scale Alfven waves (Timofeev, 1979), while (2b) describes the compressional waves.

In contrast with usual models of field line resonance, where excitation of standing Alfven waves between conjugate ionospheres is considered, here we address the generation of propagating Alfven waves, emitted from a local layer with dense plasma, but without any reflecting boundaries. Herein we consider the process of the emission of Alfven waves, partly escaping along the field lines from the conversion region, by a compressional mode confined within a layer with a low $V_{\mathrm{A}}$ (Fig. 1).

We first analyze the uncoupled Eq. (2b) without the right hand side and construct solutions describing the propagation of compressional waveguide modes along $x$ with the use of the WKB technique. The reverse influence of Alfven waves on compressional disturbances scales as $\varepsilon^{2}$ and can be neglected. In this case the expression for the potential $\psi$, which describes the modulated compressional eigenmode, has the form (with $\varepsilon$ accuracy)

$$
\psi(x, z)=A \frac{\exp \left[i \int^{x} k_{x}(\xi) d \xi\right]}{k_{x}(x)^{1 / 2} k_{\perp}(x)} w(x, z)
$$

where $A$ is the amplitude of some waveguide mode, independent of $x$ and $z$. The function $w(x, z)$ in (3) describes the distribution of the potential $\psi$ inside the waveguide in the cross-section $x=$ const (schematically shown in the bottom of Fig. 1). Both $w(x, z)$ and the transverse wave number $k_{\perp}^{2}(x)=\left(k_{x}^{2}+k_{y}^{2}\right)$ vary gradually along $x$ axis. In fact, they are just the normalized eigenfunction and eigenvalue of the operator $\hat{L}_{\mathrm{A}}(x)$, that is $\hat{L}_{\mathrm{A}}(x) w(x, z)=k_{\perp}^{2}(x) w(x, z)$. Here and throughout we assume that there is at least one propagating waveguide mode.
The region with low $V_{\mathrm{A}}$ (a "magnetic well") can be treated not only as a waveguide for a compressional mode, but also as a field-aligned resonator with opaque (partly reflecting) boundaries for Alfven waves. The partial reflection of Alfven waves takes place at the steep gradient of $V_{\mathrm{A}}$ at the walls of the magnetic well. The mechanism of partial reflection (the total reflection of an Alfven wave is impossible because the corresponding wave equation ( $2 a$ ) has no turning point) has been used already to interpret the trapping of Alfven waves in the solar chromosphere (Leroy, 1980) and the formation of an Alfven resonator in the topside ionosphere (Polyakov, 1981; Lysak, 1988).

The equation for Alfven waves emitted from a waveguide/resonator can be found by substituting the compressional potential $\psi$ into the right-hand side of (2a). Neglect of terms $\sim o\left(\epsilon^{2}\right)$ essentially simplifies the Eq. (2a), which can be reduced to an ordinary differential equation, where the coordinate $x$ comes in just as a parameter. As a result, the Alfven potential $\varphi$ can be found from the relation $u=\nabla_{\perp}^{2} \varphi$, where the function $u(x, z)$ is determined by

$$
\hat{L}_{\mathrm{A}}(x, z) u(x, z)=-i k_{y} g(x, z) \psi(x, z) .
$$

The solution of (4) provides to the first order an explicit formula for the potential $\varphi$ for Alfven waves both inside the waveguide and emitted from the resonator.

Estimates of the amplitudes of the emitted Alfven waves are expressed via the ratio $b_{\mathrm{A}}$ between the magnitude of magnetic components of an emitted Alfven wave outside the waveguide and the magnitude of a compressional mode at the center of the waveguide

$$
b_{\mathrm{A}}(x)=\left|\mathbf{B}^{(a)}(x, z= \pm \infty)\right| /\left|\mathbf{B}^{(s)}(x, z=0)\right| .
$$

The relevant magnetic fields in (5) can be determined through the expressions derived for potentials $\varphi$ and $\psi$, and (1). In Section 3 we consider a simple case when the exact analytical expressions for $b_{\mathrm{A}}(x, \omega)$ can be found.

\section{Step-like Waveguide Profile}

For illustration of the basic physics we simplify the model and assume that the field-aligned profile of Alfven velocity can be approximated by a step-like dependence on $z$ inside $\left(V_{\mathrm{A}}(|z|<a)=V_{1}\right)$ and outside $\left(V_{\mathrm{A}}(|z|>a)=V_{2}\right)$ a layer with thickness $2 a$. The variations of plasma parameters along the waveguide ( $x$-axis) are still smooth, but arbitrary. In this case the calculation of eigenvalues and eigenfunctions of the operator $\hat{L}_{\mathrm{A}}$ is similar to the problem of a rectangular potential well in quantum mechanics. Moreover, to make the expressions more tractable, we consider separately the cases when only one parameter from $V_{1}, V_{2}$ and $a$ varies along the waveguide. Explicit expressions for the dependence of the amplitude conversion factor $b_{\mathrm{A}}$ on the normalized frequency $h=\pi \omega / 2 \omega_{\mathrm{A}}$ are obtained with elementary functions for a fundamental compressional mode in two particular cases. The first one (a) is when the parameters $V_{1}$ and $V_{2}$ are fixed, but the width of the layer $2 a(x)$ varies along the waveguide, whereas the case (b) corresponds to a layer with a constant thickness, when only the Alfven velocity $V_{1}(x)$ inside a magnetic well varies. The dependences of $b_{\mathrm{A}}$ on frequency at a given fixed point $x$ have been singled out by introducing the 
dimensionless factors $b^{*}(h)$ :

$$
\begin{aligned}
& b_{\mathrm{A}}=k_{y} a\left|\frac{d a}{d x}\right| b^{*}(h), \\
& b^{*}=\left(1-\delta^{2}\right) \frac{h^{2} \cos \xi}{\left(h^{2}-\xi^{2}\right)^{3 / 2}} \cdot \frac{\delta}{\left(\tan ^{2} h+\delta^{2}\right)^{1 / 2}}, \\
& b_{\mathrm{A}}=k_{y} a\left|\frac{a d h}{h d x}\right| b^{*}(h), \\
& b^{*}=\left[\frac{\tan h}{\sin \xi}-\left(1-\delta^{2}\right)^{1 / 2}\right] \frac{h^{3} \sin 2 \xi}{\left(h^{2}-\xi^{2}\right)^{5 / 2}} \\
& \cdot \frac{\delta}{\left(\tan ^{2} h+\delta^{2}\right)^{1 / 2}} .
\end{aligned}
$$

Here the parameter $\xi(h, \delta)$ is defined implicitly by the equation $\xi(\cos \xi)^{-1}=h\left(1-\delta^{2}\right)^{1 / 2} \quad(0 \leq \xi<\pi / 2)$. The ratio $\delta=V_{1} / V_{2}$ between Alfven velocities inside and outside

case A

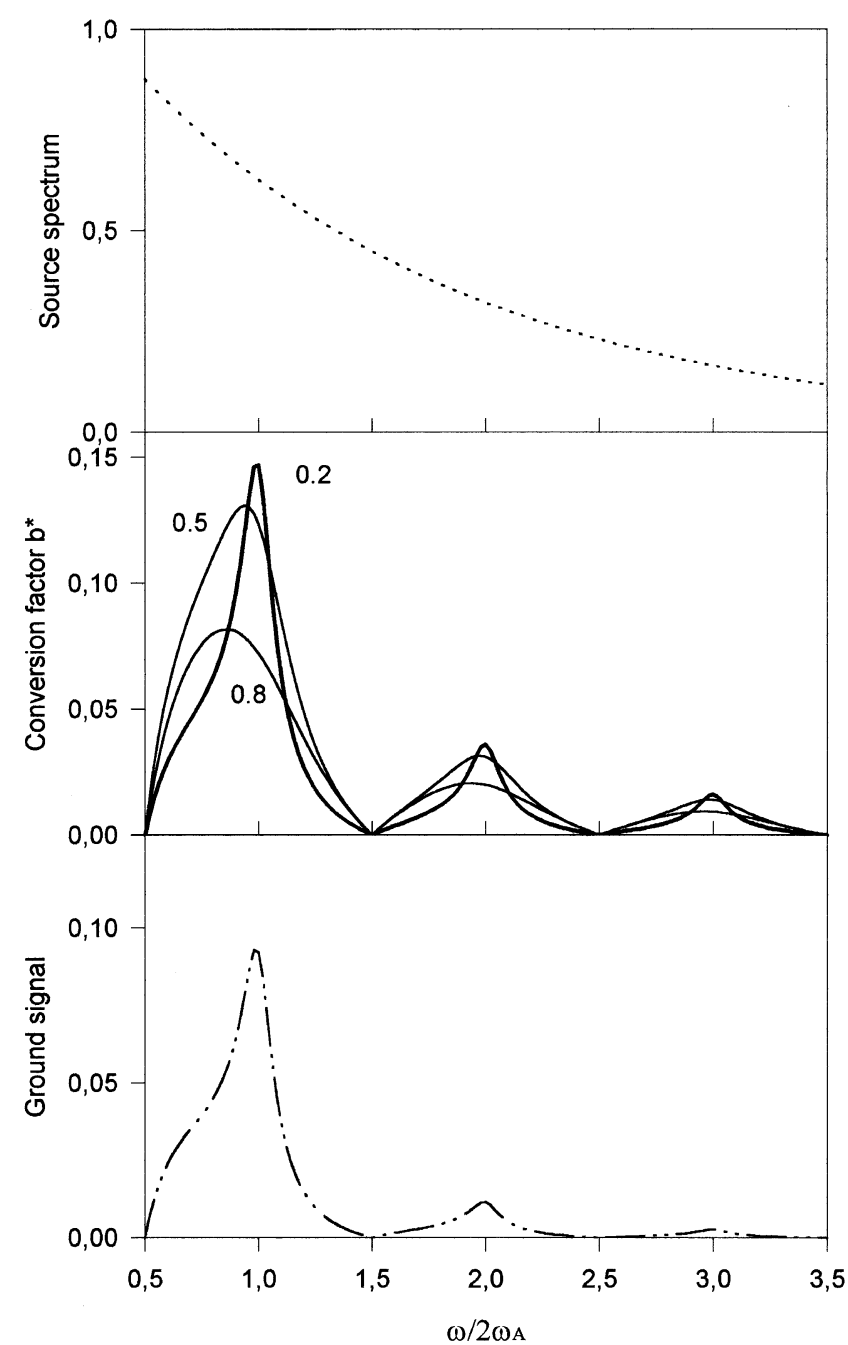

Fig. 2. Idealized color-noise spectrum in the magnetosheath in arbitrary units (upper); Dependence of the conversion factor $b^{*}$ on the normalized frequency $\omega / 2 \omega_{\mathrm{A}}$ for several values of the parameter $\delta$ for the case (a) (middle); Schematic plot of the expected spectrum (in arbitrary units) under the cusp projection (bottom). the layer, as will be shown below, determines the resonant features of the system we consider.

The general structure of the conversion factor $b^{*}(6)$ represents a function which decays with frequency, multiplied by the periodic function $\delta\left(\delta^{2}+\tan ^{2} h\right)^{-1 / 2}$ which oscillates between 0 and 1 . The dependence of $b^{*}$ in a fixed point $x$ on frequency for the case (a) is shown in the middle plot of Fig. 2 and for the case (b) in Fig. 3. In both cases these dependences are rather similar to each other.

Analysis of the expressions (6) and Figs. 2 and 3 shows that Alfven waves are excited most effectively when the frequency $\omega$ approaches the local frequencies of Alfven harmonics in the resonator, i.e. $h \rightarrow \pi n,(n=1,2, \ldots)$. The resonant nature of the transformation process is more clearly revealed in case (a). The amplitudes of resonant maxima decrease rapidly with harmonic number $n$.

The set of curves in Figs. 2 and 3, shown for different values of $\delta$, indicates that as $\delta$ decreases, the resonant spectral

\section{case B}

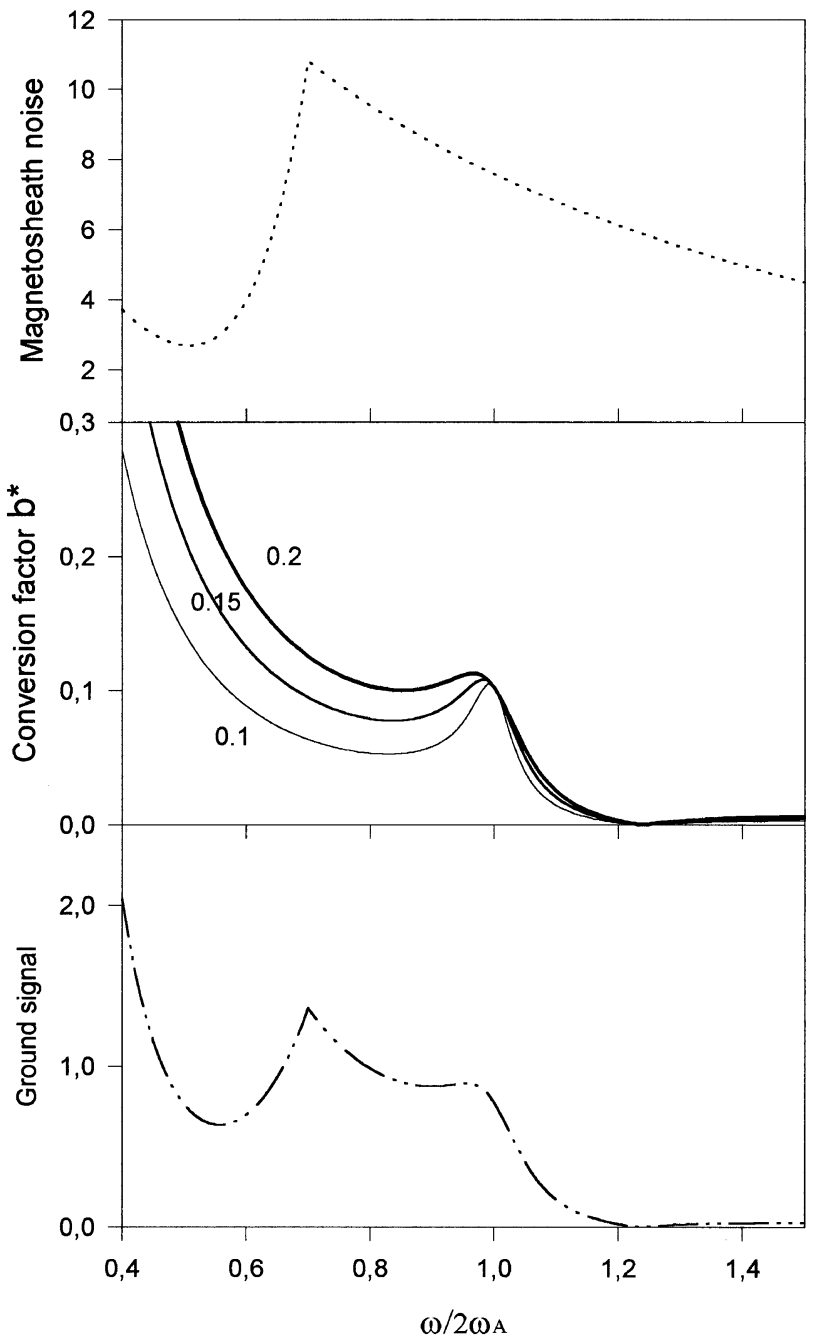

Fig. 3. Schematic view of the magnetosheath noise spectrum (in arbitrary units) with cutoff (upper); Dependence of the conversion factor $b^{*}$ on the normalized frequency for the case (b) for several values of $\delta$ (middle); Schematic plot of the expected spectrum (in arbitrary units) on the ground (bottom). 
peaks become more evident. The parameter $\delta$ determines the halfwidth of resonant peaks, e.g. $\Delta h=\delta$ or $\Delta \omega=\delta V_{1} / a$, and an effective Q-factor for an Alfven resonator, correspondingly. Summarizing the above, the resonant features of the transformation process owing to an effective Alfven resonator are given by the following

$$
\begin{array}{r}
\omega \simeq 2 n \omega_{\mathrm{A}}=\left(\pi V_{1} / a\right) n, \quad(n=1,2, \ldots), \\
Q=n \pi / 2 \delta .
\end{array}
$$

\section{Relevance to ULF Observations in the Cusp}

The high-altitude cusp in the terrestrial magnetosphere is a region where such a resonant transformation into propagating (guided) Alfven waves may take place. A local minimum of the $V_{\mathrm{A}}(z)$ distribution along the field line exists near the high-altitude cusp owing to small $B$ values. As some indirect evidence of the existence and relative stability of this high-altitude region with minimum magnetic field magnitude, we mention the observations of a "magnetic bottle" near the exterior cusp, and of its particle trapping (Chen et $a l ., 1998)$. The idea of cusp-related waveguide recently got support from the POLAR spacecraft observations (Chen and Fritz, 1998). As mentioned in this paper, the turbulent ULF magnetic energy reaches maximal magnitudes just in the region of high-altitude cusp.

This region forms an Alfven resonator without conductive boundaries. In this resonator hydromagnetic turbulence penetrating from the magnetosheath can accumulate and transform into Alfven waves escaping along the field lines. Due to the resonant nature of the transformation process a narrowband or band-limited spectrum of cusp-related $\mathrm{Pc} 3$ pulsations can be formed.

Let us consider several predictions stemming from the model of the cusp waveguide. As a rough estimate, we assume that the typical field-aligned scale of the minimum of the Alfven velocity in the region of the exterior cusp is $2 a=2 R_{\mathrm{E}}$, and that inside it, $V_{\mathrm{A}}$ drops from magnetospheric values $V_{2}=10^{3} \mathrm{~km} / \mathrm{s}$ to $V_{1}=4 \times 10^{2} \mathrm{~km} / \mathrm{s}$. Our model (7) then predicts that the most effective transformation of broadband compressional wave energy will occur at frequencies $f \simeq V_{1} / 2 a \simeq 30 \mathrm{mHz}$, which falls into the Pc3 band. The resonant features of an Alfven resonator in the cusp region can be estimated from (7), and for the above set of parameters the $Q$-factor of the fundamental mode in the cusp waveguide is $Q \simeq 4$. This value can produce experimentally noticeable modifications of the ULF spectrum. Because the heights of resonant peaks drop off sharply with harmonic number, no harmonic structure of cusp-related Pc3 waves is likely to be observed. In a schematic way the formation of a narrowband spectrum of ground Pc3 pulsations from the magnetosheath noise is depicted in Fig. 2, where in the upper plot an idealized spectrum of compressional disturbance source is shown, whereas the bottom plot shows the expected spectrum of emitted Alfven waves as a result of convolution of the source spectrum with the frequency-dependent conversion factor. According to our scheme the observed frequency would be mainly determined by local parameters of the cusp resonator.

In regard to order of magnitude, relationships (6) can be scaled as $b_{\mathrm{A}} \simeq b^{*}\left(k_{y} a\right)\left(a / L_{x}\right)$, where $L_{x}=(\partial \log a / \partial x)^{-1}$ is the scale of the waveguide inhomogeneity. Formally, the model is valid under the approximation $\left(a / L_{x}\right) \ll 1$ due to the assumed smoothness of variations of plasma parameters. However, the estimates obtained under a WKB approximation are qualitatively valid even at the margin of applicability, i.e., when $\left(a / L_{x}\right) \leq 1$. The azimuthal component of the wave vector $k_{y}$ is approximately the inverse of the azimuthal scale of the cusp, that is $k_{y} \simeq L_{y}^{-1} \simeq 1 / 2 R_{\mathrm{E}}$. Thus, the above estimates show that emitted Alfven waves may reach amplitudes about 1 to 1.5 orders of magnitude less than the amplitudes of the driving compressional wave activity. The ground ULF signature from Alfven waves may be higher, by a factor $\sqrt{B_{\text {ionosphere }} / B_{\text {cusp }}} \sim 10$ owing to convergence of geomagnetic field lines. Note also that relationships (6) describe amplitudes of emitted Alfven waves outside the resonator. Amplitudes of Alfven waves trapped within the resonator are about $\delta^{-1}$ times larger.

The recent observations by POLAR show that in the dayside polar cusp a local magnetic turbulence demonstrates several spectral peaks near $0.3 \mathrm{~Hz}$ (see figure 4 in Chen and Fritz (1998)) with power density up to $\sim 10^{3} \mathrm{nT}^{2} / \mathrm{Hz}$. Even with relatively low conversion rates given by the WKB approximation, the cusp turbulence of such intensity may ensure ground ULF signals of order of several nT.

As (6) demonstrates, the transformation efficiency grows when the gradient becomes more steep. The realistic physical situation may well correspond to the case $a / L_{x} \simeq 1$, which is too difficult for the analytical treatment. So, it would be desirable to consider analytically the other extreme situation, when $a / L_{x} \gg 1$, and to check the match between the results of two models at the boundary of validity. The most efficient way the predictions of our model can be verified is with the use of modern 2- and 3-dimensional MHD codes, which have been used so far only for the study of the propagation/transformation of compressional pulses launched at the magnetospheric equator (Allan et al., 1986; Lee and Lysak, 1989).

The decaying behavior of the dependence $b_{\mathrm{A}}(\omega)$, especially evident in case (b), indicates that the high-latitude Alfven resonator in the cusp can operate also as a low-pass filter. Examination of characteristics of the broadband magnetosheath noise spectra (e.g., Plates 2 and 4 of Engebretson et al., 1991b) indicates a low-frequency cutoff, as schematically shown in the upper plot of Fig. 3. This cutoff frequency corresponds to the upstream wave frequency, in general. Hence, the band-limited ULF spectral structure observed in cusp-latitude ground magnetometer data, as depicted in the bottom plot of Fig. 3, can be produced by the combination of two effects: a low-frequency cutoff characteristic of the magnetosheath noise and low-pass features of the transformation process. In this case the frequency of this non-resonant spectral peak is mainly determined by the cutoff frequency, controlled by IMF magnitude.

The growth of $b^{*}$ at low frequencies $\omega \ll \omega_{\mathrm{A}}$, evident in Fig. 3, is related to the manifestation of a surface-type compressional mode, guided by the thin plasma layer. The feature of this mode is a large inertial current $j_{x}$. The noncompensating part of $j_{x}$ in an inhomogeneous plasma produces large field-aligned currents. However, the occurrence 
of the guided surface-type compressional mode is just a formal consequence of the model we have considered and it cannot be observed in reality. In fact, its spatial scale exceeds the size of the MHD waveguide in the cusp. Thus, this model cannot be applied for the ULF cusp phenomena in the low-frequency range $\omega \ll \omega_{\mathrm{A}}$, though it may describe guidance of compressional disturbances along a large-scale plasma layer, like in the magnetosphere of Saturn (Cramm et al., 1998).

The model developed is based on a variation of the Alfven velocity that is due to the variation in plasma density $N$, whereas in the real situation the variation is primarily due to the variability in the magnitude of the magnetic field $B$. Actually, for the same geometry of the magnetic field, MHD modes are sensitive to variations of Alfven velocity, but not to separate variations of $N$ or $B$. So, the model with an inhomogeneous plasma density only is a good approximation of the real situation.

The model assumes that the variations of the Alfven velocity is symmetric around $z=0$, because the comprehensive analytical treatment of non-symmetric profile of Alfven velocity (e.g., $V_{\mathrm{A}}(-\infty) \neq V_{\mathrm{A}}(+\infty)$ ) is much more cumbersome. When this simplifying assumption is not valid, we expect that principal features of the waveguide/resonator remain qualitatively the same, but the emission of Alfven waves would be non-symmetric: the leakage into a side with smaller gradient of $V_{A}$ would be higher.

\section{Discussion}

In this paper we have considered only one aspect of a more general problem: what are the physical differences between cusp-associated Pc3 and mid-latitude Pc3 pulsations? Are these different classes of MHD activity, just accidentally falling in the same frequency band? Which is the main channel of transmission of wave energy in the Pc3 band from the solar wind into the magnetosphere-through the cusp or through the near-equatorial magnetosphere? Earlier analyses of the meridional distribution of Pc3 pulsations (Bol'shakova and Troitskaya, 1984; Plyasova-Bakounina et al., 1986) noted a maximum at cusp latitudes. However, these studies compared only averaged amplitudes over about 10-min and longer intervals, and consequently these results only indicate that the intensity of waves in the Pc3 band in the cusp is higher than at mid-latitudes. They do not address the question of the possible identity (a common source) of the signals.

We are inclined to think that the cusp Pc3 and mid-latitude Pc3 have a common source in turbulent magnetosheath plasma, the features of which depend predominantly on IMF orientation, but that their means of penetration to the ionosphere are different. One channel is the propagation of compressional mode waves in the near-equatorial magnetosphere. There, periodic wave trains are formed under the influence of magnetospheric field line Alfven resonances. Another channel is the penetration of magnetosheath wave turbulence directly via the polar cusp region. Here also some filtering of input signals takes place, and in this paper we have indicated one possible mechanism. Hence, we expect that general characteristics of Pc3 activity at the cusp latitudes and at mid-latitudes might be similar, because they are caused by the same source. However, because the propagation channels are different, we would not expect to find a detailed coherence between cusp Pc3 and mid-latitude Pc3 activity: the difference in the channels of the penetration of wave energy into the magnetosphere through the cusp and via the equatorial plane of the magnetosphere results in the absence of close correlations between the separate daytime Pc3 packets at high and middle latitudes (Pilipenko et al., 1996).

It is also probable that some non-MHD plasma mechanisms operating in the cusp, such as flow and current instabilities, modulated particle precipitation, ionospheric turbulence, etc., produce magnetic signals in the same ULF band. However, most of these models actually predict the generation of wide-band turbulence.

The analysis of possible plasma parameters near the high altitude cusp shows that resonant features of the MHD waveguide/resonator are not very high, which may explain that narrow-band Pc3 are relatively rare at cusp latitudes as compared with Pi1. The resonant features of the cusp-related resonator depend on the actual field-aligned profile of an Alfven velocity, and when the effective $Q$-factor drops to unity and less, the band-limited resonant filtering is not well pronounced and broad-band wave activity are to be observed.

According to our scheme the band-limited filtering of magnetosheath noise may occur owing to resonant features of the wave conversion mechanism (Fig. 2). In this case an observed wave packet's frequency is mainly determined by local parameters of the cusp resonator, which may explain the absence of Pc3-IMF correlations at cusp latitudes below 30 $\mathrm{mHz}$ in the data set of Wolfe et al. (1990). There is also a possibility that band-limited spectral structure of Pc3 is formed in non-resonant way, as a combination of cutoff frequency in the magnetosheath spectrum and decaying frequency dependence of the wave conversion efficiency (Fig. 3). In this case an expected frequency is related to the cutoff frequency, which is controlled by the IMF magnitude.

Below we will indicate briefly the expected distinctions between cusp-associated narrow-band Pc3 and mid-latitude Pc3. First, due to the specific polarization asymmetry of resonant amplification of field line oscillations at mid-latitudes, the $H$ component is enhanced in comparison to the $D$ component. This fact is already used for the extraction of the resonant frequency from complicated ULF spectra in the socalled $H / D$ polarization method (Baransky et al., 1995). A similar asymmetry is not expected to be observed for the cusp-associated Pc3.

Our suggested mechanism has a second geophysical consequence which differentiates the excitation of standing field line oscillations from the generation of propagating Alfven wavelets. In the former case a good conjugacy between ULF waves in opposite ionospheres should be observed at cusp latitudes. The latter scenario, on the contrary, implies a lack of coherence between conjugate points. This conclusion stems from the following qualitative consideration. During propagation the front of an Alfven pulse undergoes distortion owing to the inhomogeneous distribution of $V_{\mathrm{A}}$ across a field line. In other words, the transverse effective wave vector $k_{x}$ steadily grows with distance $z$ along a field line, as $k_{x}(z) \simeq k_{x}(0)+\left(\partial \omega_{\mathrm{A}} / \partial x\right)\left(z / V_{\mathrm{A}}\right)$. This effect is anal- 
ogous to the phase mixing during the time evolution of an Alfven disturbance in an inhomogeneous system. As a result, when an Alfven pulse reaches the ionosphere its transverse wave vector will be $k_{x} \simeq L_{x}^{-1}\left(k_{\|} l\right)$, where $L_{x}$ is the scale of the transverse inhomogeneity, and $l$ is the distance passed. For example, an Alfven pulse emitted from the northern polar cusp will have a scale above the southern ionosphere $k_{x} \simeq 2 \times 10^{-2} \mathrm{~km}^{-1}$ for $k_{\|} l \simeq 10$ and $L_{x} \simeq 5 \times 10^{2} \mathrm{~km}$. This signal is substantially attenuated at the ground, because $k_{x} H \simeq 2$ ( $H \simeq 120 \mathrm{~km}$ is the height of the ionosphere). Upon reflection from the ionosphere an Alfven pulse will get an additional increase of transverse wave vector of about $\Delta k_{x} \simeq 2 k_{\|} \cot I$ ( $I$ is the inclination of the geomagnetic field), which will further decrease the transverse scale. The above relationship follows from the conservation of the horizontal component of the Alfven wave vector upon reflection from the ionosphere. As a result the Pc3 signal is likely to totally dissipate due to small-scale kinetic mechanisms on its return flight. Hence, although the Alfven pulses may be emitted symmetrically from the polar cusp waveguide in both directions along the field lines, the signal at the conjugate point will be substantially attenuated due to phase mixing over its larger travel distance, even in the absence of any damping mechanisms. So, while overall ULF activity in both cusps, primarily determined by the turbulence level in the solar wind, might be similar in general, we would expect to find no coherence between separate wave packets.

This consequence of our model is in accord with some cusp-related ULF observations. Analysis of Pc3 pulsations at the conjugate stations Iqaluit, Canada and South Pole, Antarctica by Wolfe et al. (1990) during local daytime hours indicated a lack of conjugacy on an event by event basis.

We suggest also a third distinctive feature of cusp-related Pc3 activity. Mid-latitude Pc3 waves are believed to be transported from an extra-magnetospheric source by large-scale compressional waves, which ensures the global nature of these pulsations. They have large scales in the azimuthal direction (or small $m$ values). Though the meridional distribution of Pc3 amplitudes might be non-monotonic, the Pc3 wave packets can be traced from sub-auroral latitudes to the equator. On the contrary, the proposed model of cusp Pc3 assumes the excitation of Alfven waves in a relatively localized region of the magnetosphere. As was mentioned above, Alfven waves are weakly converted into compressional waves. Thus, we expect that the cusp-related Pc3 would be a localized wave phenomenon, mostly associated with the equatorward cusp boundary.

Some observational results confirm this notion. Alford et al. (1996) studied the spatial structure of relatively narrowband Pc3-4 signals near local noon at the MACCS network in Arctic Canada, covering 5 hours in magnetic LT at latitudes from $75^{\circ}$ to $79^{\circ}$. Multistation comparisons showed that the spatial scale of Pc3 pulsations was typically less than $300 \mathrm{~km}$. This result is consistent with the observations reported by Olson and Fraser (1994), who found good coherence only between two cusp-latitude stations separated by $150 \mathrm{~km}$. Similarly, Olson and Szuberla (1997) and Szuberla et al. (1998), though not discriminating clearly between Pi1 and Pc3, estimated the upper bound for the coherence length of these pulsations near the cusp to be near or slightly less than $200 \mathrm{~km}$. The scale of an Alfven wave beam in the ionosphere must be about $\sim 120 \mathrm{~km}$ less than at the ground. Thus the ground magnetic observations fit the Halley PACE radar observations, indicating lack of coherence at Pc3 frequencies over distances in the North-South direction beyond about 60 km (Baker et al., 1998).

\section{Conclusion}

The existence of new magnetospheric phenomenon: MHD waveguide/resonator in the high-altitude cusp is suggested. The accumulation of turbulent ULF magnetic energy in the region of the high-altitude cusp evidenced by POLAR spacecraft observations is consistent with this prediction. However, a relevant analytical description of the wave trapping and transformation in such a system is actually a complicated 2-dimensional problem, which is much beyond the standard 1-dimensional field line resonance theory. The analytical model developed, though having some pitfalls caused by oversimplifications of the realistic situation, predicts leakage of Alfven waves from the region with trapped wave energy. In contrast to the well-known model of field line resonance, in the mechanism suggested here the transformation of ULF wave modes occurs in a localized region with a decreased Alfven velocity, which has no reflecting boundaries. This transformation into propagating Alfven waves has a frequency-resonant character, which may ensure the formation of band-limited spectra of ULF Pc3 pulsations in the cusp region. Also, it is possible that band-limited Pc3 spectra could be the result of the convolution of the typical magnetosheath spectrum with cutoff frequency with the frequency decaying conversion factor.

Probably, the cusp-associated narrow-band Pc3 waves and mid-latitude Pc3 waves have a common source in the turbulent magnetosheath plasma, the features of which depend on the orientation and magnitude of the IMF. However, their mechanism of penetration to the ionosphere is different, and it is this which enables us to consider them as different wave phenomena. Observations with a dense meridional profile with sufficiently high time resolution will be needed to confirm this mechanism and determine the spatial boundary between cusp-related Pc3/Pi1 and mid-latitude Pc3 waves.

Acknowledgments. The collaboration of the Institute of the Physics of the Earth with Augsburg College and Boston University is supported by National Science Foundation grants ATM-9610072 to Augsburg College and ATM-9704766 to Boston University.

\section{References}

Alford, J. L., M. J. Engebretson, K. A. Schmidt, W. J. Hughes, R. L. Arnoldy, C. G. Maclennan, and H. Fukunishi, Studies of the coherence and propagation of Pc 3 pulsations at cusp/cleft/polar cap latitudes (abstract), EOS Trans. AGU, 77, 46, Fall Meet. Suppl, F609, 1996.

Allan, W., S. P. White, and E. M. Poulter, Impulse excited hydromagnetic cavity and field-line resonances in the magnetosphere, Planet. Space Sci. 34, 371-385, 1986.

Baker, K. B., M. J. Engebretson, A. S. Rodger, and R. L. Arnoldy, The coherence scale length of band-limited $\mathrm{Pc} 3$ pulsations in the ionosphere, Geophys. Res. Lett., 25, 2357-2360, 1998.

Baransky, L. N., A. W. Green, E. N. Fedorov, N. A. Kurneva, V. A. Pilipenko, and W. Worthington, Gradient and polarization methods of ground-based monitoring of magnetospheric plasma, J. Geomag. Geoelectr., 47, 12931309, 1995.

Bol'shakova, O. V. and V. A. Troitskaya, The relation of the high-latitude maximum of Pc 3 intensity to the dayside cusp, Geomagn. Aeron., Engl. 
Transl., 24, 633-636, 1984.

Chen, J., T. A. Fritz, R. B. Sheldon, H. E. Spence, W. N. Spjeldvik, J. F. Fennell, S. Livi, C. T. Russell, J. S. Pickett., and D. A. Gurnett, Cusp energetic particle events: Implications for a major acceleration region of the magnetosphere, J. Geophys. Res., 103, 69-78, 1998.

Chen, J. and T. A. Fritz, Correlation of cusp MeV helium with turbulent ULF power spectra and its implications, Geophys. Res. Lett., 25, 4113-4116, 1998.

Cramm, R., K.-H. Glassmeier, M. Stellmacher, and C. Othmer, Evidence for resonant mode coupling in Saturn's magnetosphere, J. Geophys. Res., 103, 11,951-11,960, 1998.

Engebretson M. J., C.-I. Meng, R. L. Arnoldy, and L. J. Cahill, Jr., Pc3 pulsations observed near the south polar cusp, Geophys. Res. Lett., 91, 8909-8918, 1986.

Engebretson, M. J., B. J. Anderson, L. J. Cahill, Jr., R. L. Arnoldy, P. T. Newell, C.-I. Meng, L. J. Zanetti, and T. A. Potemra, A multipoint case study of high latitude daytime ULF pulsations, Geophys. Res. Lett., 94, 17,143-17,160, 1989.

Engebretson, M. J., L. J. Cahill, Jr., R. L. Arnoldy, B. J. Anderson, T. J. Rosenberg, D. L. Carpenter, U. S. Inan, and R. H. Eather, The role of the ionosphere in coupling upstream ULF wave power into the dayside magnetosphere, Geophys. Res. Lett., 96, 1527-1542, 1991a.

Engebretson, M. J., N. Lin, W. Baumjohann, H. Luehr, B. J. Anderson, L. J. Zanetti, T. A. Potemra, R. L. McPherron, and M. G. Kivelson, A comparison of ULF fluctuations in the solar wind, magnetosheath, and dayside magnetosphere, 1. Magnetosheath morphology, Geophys. Res. Lett., 96, 3441-3454, 1991b.

Engebretson, M. J., J. R. Beck, D. L. Detrick, T. J. Rosenberg, R. L. Rairden, S. B. Mende, R. L. Arnoldy, and L. J. Cahill, Jr., Optical evidence that modulated electron precipitation near the magnetospheric boundary drives high latitude Pc3-4 magnetic pulsations, in Physical Signatures of Magnetospheric Boundary Layer Processes, NATO ASI Series C, vol. 425, edited by J. A. Holtet, and A. Egeland, 456 pp., pp. 361-373, Kluwer, Dordrecht, the Netherlands, 1994.

Engebretson, M. J., W. J. Hughes, J. L. Alford, E. Zesta, L. J. Cahill, Jr., R. L. Arnoldy, and G. D. Reeves, Magnetometer array for cusp and cleft studies observations of the spatial extent of broadband ULF magnetic pulsations of cusp/cleft latitudes, J. Geophys. Res., 100, 19,371-19,386, 1995.

Fedorov, E. N., N. G. Mazur, V. A. Pilipenko, and K. Yumoto, On the theory of field line resonances in plasma configurations, Physics of Plasmas, 2(2), 527-532, 1995

Fedorov, E. N., N. G. Mazur, V. A. Pilipenko, and K. Yumoto, MHD wave conversion in plasma waveguides, J. Geophys. Res., 103, 26,595-26,605, 1998.

Glassmeier, K.-H., ULF pulsations in the polar cusp and cap, in Electromagnetic Coupling in the Polar Clefts and Caps, NATO ASI Series, edited by P. E. Sandholt and A. Egeland, 465 pp., pp. 167-186, Kluwer, Dordrecht, the Netherlands, 1989.

Le, G. and C. T. Russell, The morphology of ULF waves in the Earth's foreshock, in Solar Wind Sources of Magnetospheric ULF Waves, Geophysical Monograph 81, edited by M. J. Engebretson, K. Takahashi, and M. Scholer, 424 pp., pp. 87-98, AGU, Washington D.C., 1994.
Lee, D.-H. and R. L. Lysak, Magnetospheric ULF wave coupling in the dipole model: the impulsive excitation, J. Geophys. Res., 94, 17,097$17,104,1989$

Lepidi, S., U. Villante, M. Vellante, P. Palangio, and A. Meloni, High resolution geomagnetic field observations at Terra Nova Bay, Antarctica, Annali di Geofizica, 33, 519-525, 1996.

Leroy, B., Propagation of waves in an atmosphere in the presence of a magnetic field. II. The reflection of Alfven waves, Astron. Astrophys. 91, 136-146, 1980.

Lin, N., M. J. Engebretson, R. L. McPherron, M. G. Kivelson, W. Baujohann, H. Luehr, T. A. Potemra, B. J. Anderson, and L. J. Zanetti, A comparison of ULF fluctuations in the solar wind, magnetosheath, and dayside magnetosphere, 2. Field and plasma conditions in the magnetosheath, $J$. Geophys. Res., 96, 3455-3464, 1991.

Lysak, R. L., Theory of auroral zone Pib pulsation spectra, J. Geophys. Res. 93, 5942-5946, 1988 .

Olson, J. V., ULF signatures of the polar cusp, J. Geophys. Res., 91, 10,055$10,062,1986$

Olson, J. V. and B. J. Fraser, Pc3 pulsations in the cusp, in Solar Wind Sources of Magnetospheric ULF Waves, Geophysical Monograph 81, edited by M. J. Engebretson, K. Takahashi, and M. Scholer, 424 pp., pp. 325-334, AGU, Washington D.C., 1994.

Olson, J. V. and C. A. L. Szuberla, A study of Pc3 coherence at cusp latitudes, J. Geophys. Res., 102, 11,375-11,383, 1997.

Pilipenko, V., N. Kleimenova, O. Kozyreva, K. Yumoto, and J. Bitterly, Is the cusp a source of mid-latitude Pc3 pulsations? Geomagn. and Aeronomy, 36, 39-48, 1996.

Plyasova-Bakounina, T. A., V. A. Troitskaya, J. W. Munch, and H. F. Gauler, Super-high-latitude maximum of Pc2-4 intensity, Acta Geod., Geophys. et Montanist. Hung., 21, 143-153, 1986.

Polyakov, S. V., Ionospheric Alfven resonator, Geomagn. and Aeronomy, 21, 371-377, 1981

Southwood, D. J., Some features of field line resonances in the magnetosphere, Planet. Space Sci., 22, 483-491, 1974

Szuberla, C. A. L., J. V. Olson, M. J. Engebretson, B. J. Fraser, S. Ables, and W. J. Hughes, Interstation Pc3 coherence at cusp latitudes, Geophys. Res. Lett., 25, 2381-2384, 1998.

Timofeev, A. V., To the theory of Alfven oscillations in an inhomogeneous plasma, in Reviews of Plasma Physics, Vol. 9, edited by M. A. Leontovich, pp. 205-232, Plenum Publishing Corp., 1979.

Wolfe, A., D. Venkatesan, R. Slawinski, and C. G. Maclennan, A conjugate area study of Pc3 pulsations near cusp latitudes, J. Geophys. Res., 95 , 10,695-10,698, 1990.

Zhang, X., H. Comfort, Z. E. Musielak, T. E. Moore, D. L. Gallagher, and J. L. Green, Propagation characteristics of Pc3 compressional waves generated at the dayside magnetopause, J. Geophys. Res., 98, 15,403$15,410,1993$

V. Pilipenko (e-mail: vpilipenko@uipe-ras.scgis.ru), E. Fedorov, N. Mazur, M. J. Engebretson (e-mail: engebret@augsburg.edu), and W. J. Hughes (e-mail: hughes@buasta.bu.edu) 